\title{
Autoantibodies spectrum in lupus nephritis in a cohort of Egyptian patients: relation to disease activity and prognostic value
}

\author{
Sahar A. Elsayed ${ }^{1 *}$ iD and Omar M M. Mohafez ${ }^{2}$
}

\begin{abstract}
Background: Specific autoantibodies are considered as an important marker in autoimmune rheumatic diseases and are of great value for the diagnosis and prognosis of systemic lupus erythematosus (SLE) patients. A total of eighteen autoantibodies were analyzed for their positivity in SLE patients and we evaluated the clinical relevance of the five most frequent autoantibodies: anti-dsDNA, anti-nucleosome, anti-histone, anti-Ro60, and anti-Ro52 on disease activity and renal affection in SLE Egyptian patients.

Results: Immunological profile and correlation of the five autoantibodies with disease activity and histopathological pattern of renal involvement were analyzed for 190 SLE patients. Lupus nephritis (LN) patients showed much worse constitutional and mucocutaneous manifestations than patients without nephritis. Autoantibody profile showed a significant increased frequency of anti-dsDNA, anti-nucleosome, anti-histone, anti-Ro-60, and anti-Ro52 antibodies in LN patients. The impact of the co-positivity of the autoantibodies on the renal function was obvious. Moreover, the disease activity increased by the increased frequency of autoantibodies positivity in LN patients. ROC curve analysis showed that anti-nucleosome had the highest sensitivity; $93 \%$ followed by anti-dsDNA $83.3 \%$ then anti-histone $73.8 \%$, but anti-Ro60 and anti-Ro52 showed a humble sensitivity. Furthermore, the highest frequency of positivity for the five autoantibodies was found in class-III and class-IV LN patients.

Conclusion: Detection of anti-dsDNA, anti-nucleosome, anti-histone, and anti-Ro60 in SLE patients may be important for predicting disease progression and kidney affection. Moreover, anti-nucleosome and anti-dsDNA show high sensitivity and specificity for lupus nephritis, thus patients with four to five positive autoantibody panels should be kept under close monitoring as they may warrant considering aggressive therapy to control their disease and prevent renal damage.
\end{abstract}

Keywords: Autoantibodies, Lupus nephritis, ANA profile, SLE

Message: Autoantibodies serve as diagnostic, prognostic, and activity markers for SLE

\section{Background}

Systemic lupus erythematosus (SLE) is an autoimmune disease characterized by a dysregulated immune system and manifested by the production of excessive pathogenic autoantibodies, which have a diagnostic application and are implicated in organ involvement [1]. Lupus

\footnotetext{
* Correspondence: saharomar2000@yahoo.co.uk

'Department of Rheumatology and Rehabilitation, Faculty of Medicine,

Sohag University, Sohag 82524, Egypt

Full list of author information is available at the end of the article
}

nephritis (LN) is one of the most common causes of mortality and morbidity. SLE patients with LN have about sixfold higher mortality rates than those without LN [2]. Over the recent few decades, the 10-year survival rate of LN has dramatically improved from 46 to $95 \%$ among patients who achieved disease remission [3]. Nonetheless, around $5-20 \%$ of lupus nephritis patients will develop end-stage renal disease within 10 years after being diagnosed despite receiving aggressive immunosuppressive therapy [4].

\section{Springer Open}

(ㅇ The Author(s). 2020 Open Access This article is licensed under a Creative Commons Attribution 4.0 International License, which permits use, sharing, adaptation, distribution and reproduction in any medium or format, as long as you give appropriate credit to the original author(s) and the source, provide a link to the Creative Commons licence, and indicate if changes were made. The images or other third party material in this article are included in the article's Creative Commons licence, unless indicated otherwise in a credit line to the material. If material is not included in the article's Creative Commons licence and your intended use is not permitted by statutory regulation or exceeds the permitted use, you will need to obtain permission directly from the copyright holder. To view a copy of this licence, visit http://creativecommons.org/licenses/by/4.0/. 
Autoantibodies for vital components including nucleic acids, phospholipids, proteins, glycoproteins, nucleoproteins, and glycolipids are an important finding in autoimmune rheumatic diseases. Antinuclear antibodies (ANA) are a group of autoantibodies that target nuclear antigens. Most patients with SLE have positive ANA but not all, ANA positive individuals have lupus as it may be present in other diseases and in elderly individuals [5]. Detection of a specific autoantibody can be beneficial in the diagnosis of certain autoimmune diseases. For instance, ANA, anti-Smith (anti-Sm), and anti-doublestranded DNA (anti-dsDNA) antibodies are part of the American College of Rheumatology criteria for the diagnosis of SLE [6]. ANA by indirect immunofluorescence assay (IIF) with a cutoff titer $1 / 80$ is highly sensitive and specific for SLE [7]. Although, anti-dsDNA is suggested to be a good marker for the diagnosis of SLE and monitoring disease activity, however, anti-nucleosome is reported to provide a better indicator for disease activity $[8,9]$. Also, a good association is reported between antinucleosome and LN [10]. Previous studies revealed conflicting results regarding anti-histone considering it as a specific marker for the diagnosis of SLE with a comparable diagnostic value to anti-dsDNA while other studies revealed that it is sensitive but not specific for SLE [11]. Among the autoantibodies which have been detected in the sera of patients with autoimmune disease are antiRo60 (anti-SSA) and anti-Ro52 (TRIM21) [12]. Ro52 and Ro60 are proteins with a molecular weight of 52 and $60 \mathrm{kDa}$ respectively, having different biochemical and immunological functions [13]. Ro52 is involved in protein ubiquitination and apoptosis while Ro60 is a component of small cytoplasmic ribonucleoprotein complex, which plays a role in the degradation of misfolded noncoding ribonucleic acid (RNA). Anti-Ro60 and antiRo52 are commonly detected in a variety of autoimmune diseases and are associated with various clinical manifestations [14].

Despite previous studies demonstrated that certain ANA is associated with a variety of clinical manifestations in SLE but little data is available showing the role of individual ANA profile in the development and progression of $\mathrm{LN}$ and almost no data are available showing the association between the most used antibodies: antidsDNA, anti-nucleosome, anti-histone, anti-Ro-60 and anti-Ro52, and nephritis. So, we aimed to evaluate their clinical relevance on disease activity and to clarify their correlation with renal affection in SLE patients.

\section{Methods}

In this study, the data for 190 adult SLE patients were collected from the department of rheumatology, from September 2018 to February 2020. Our patients were diagnosed according to the 1982 ACR revised criteria for
SLE [15]. Patients with concomitant systemic diseases and other autoimmune diseases including rheumatoid arthritis, scleroderma, mixed connective tissue disease, Sjogren's syndrome, polymyositis, pregnant females, and other causes of nephritis were excluded. Patients were classified into two groups based on the presence or absence of biopsy proved LN. The first group included 102 patients with LN; 93 females (91.18\%) and 9 males $(8.82 \%)$ and the second group included 88 patients without LN; 81 females (92.05\%) and 7males (7.95\%). $\mathrm{CBC}$ and total protein were done for all patients as a routine lab.

Renal biopsy, histopathology, and classification were determined in the department of pathology according to the International Society of Nephrology/Renal Pathology Society (ISN/RPS). Renal parameters were assessed in the form of urinary sediment, casts, serum creatinine, blood urea, and protein/creatinine $(\mathrm{P} / \mathrm{C})$ ratio. Assessment of the disease activity in the patients was done using the SLE disease activity index (SLEDAI). No activity: 0 , mild activity: $1-5$, moderate activity: $6-10$, high activity: 11-19, very high > 20 [16].

ANA was done using indirect immunofluorescence assay (IIF) on HEp-2 cell. Using ANAFLUOR, DiaSorin, Stillwater, Minnesota 55082-0285, USA kit according to the manufacturing protocol. Briefly, $20 \mu \mathrm{l}$ of diluted serum was incubated with fixed HEp-2 cell slide for 30 min. After wash, $20 \mu \mathrm{l}$ of antihuman immunoglobulin conjugated with fluorescein isothiocyanate (FITC) was added and incubated for an additional $30 \mathrm{~min}$ then washed and a drop of mounting media was applied, and the slides were read using a fluorescence microscope [17].

ANA profile was done using dot immunoassay by automated enzyme immunoassay EIA: A commercially available dot immunoassay was performed by using BlueDiver Quantrix ANA19 IgG kit (Code: ANA19Q24) for BlueDiver Instrument (BDI), (both D-tek, Rue René Descartes 19, 7000 Mons-Belgium) used for the detection of IgG autoantibodies against dsDNA, nucleosome, histones, Ro52, Ro60, Smith, RNP, La, Scl-70, RNA polymerase, Ku, PM-Scl100, SmRNP, Mi-2, Jo-1, CENP-A, PCNA, and ribosome antigens in human sera. The test principle is based on a classical enzyme immunoassay (EIA), according to the manufactural protocol [18].

\section{Statistical analysis}

(SPSS), version 20 (IBM Corp., Armonk, USA) was used for the data analysis. Qualitative data were represented as numbers and percentages. We used KolmogorovSmirnov test to check the normal distribution of data. Data and results were expressed in mean with standard deviation in normally distributed data. The Kendall's 
tau-b correlation was used for correlation between autoantibodies in the ANA profile. Independent-sample $t$ test was used for normally distributed variables. We used the Kruskal-Wallis test to compare three or more groups then Mann-Whitney $U$ test was used to compare two groups for not normally distributed data. The receiver operating curve (ROC) was done by plotting sensitivity on the $y$-axis and 1 -specificity on the $x$-axis. The area under the curve (AUC) is considered poor for 0.50-0.60, fair for $0.70-0.80$, good for $0.8-0.9$, and $0.90-1$ is considered excellent [19].

\section{Results}

Our study included 190 adult SLE patients (102 with nephritis and 88 without nephritis). The demographic, clinical, laboratory findings and medication characteristics of the patients are shown in Table 1. Constitutional, mucocutaneous, and musculoskeletal manifestations were significantly much worse in lupus nephritis patients in comparison to patients without nephritis. Serum creatinine and $\mathrm{P} / \mathrm{C}$ ratio were significantly higher in $\mathrm{LN}$ patients. On the other hand, hemoglobin was significantly lower in LN. In regard to disease activity, as shown in Fig. 1a, the majority of patients without nephritis were distributed in the mild and moderate disease activity groups whereas only $6.8 \%$ were in the high disease activity group. However, $23.3 \%$ of patients with nephritis showed high disease activity.

ANA profile in SLE patients with and without nephritis A total of eighteen autoantibodies were analyzed for their positivity in SLE patients as shown in Table 2 .

Table 1 Demographic and clinical characteristics of SLE patients

\begin{tabular}{|c|c|c|c|}
\hline & \multicolumn{3}{|l|}{ SLE patients } \\
\hline & With nephritis $(n=102)$ & Without nephritis $(n=88)$ & $p$ value \\
\hline Female: Male ratio & $93(91.18 \%) / 9$ (8.82\%) & 81 (92.05\%)/7 (7.95\%) & 0.824 \\
\hline Age (years) & 31.49 (18.61-66.43) & $35.25(18.15-65.71)$ & 0.622 \\
\hline Family history & $4(3.92 \%)$ & $1(1.13 \%)$ & 0.180 \\
\hline Disease duration (years) & $3.69(0.41-17.50)$ & $4.57(0.16-18.00)$ & 0.739 \\
\hline \multicolumn{4}{|l|}{ Clinical manifestations } \\
\hline Constitutional & $87(85.29 \%)$ & $55(62.5 \%)$ & 0.011 \\
\hline Mucocutaneous & $82(80.39 \%)$ & $28(31.81 \%)$ & $<0.001$ \\
\hline Musculoskeletal & 91 (89.2\%) & $30(34.09 \%)$ & $<0.001$ \\
\hline Lupus nephritis & $102(100 \%)$ & $0(0 \%)$ & \\
\hline Cardiovascular & $6(5.88 \%)$ & $3(3.4 \%)$ & 0.527 \\
\hline Pulmonary & $3(2.94 \%)$ & $1(1.13 \%)$ & 0.317 \\
\hline Neuropsychiatric & $9(8.82 \%)$ & $5(5.68 \%)$ & 0.439 \\
\hline Hematological & $92(90.19 \%)$ & $70(79.54 \%)$ & 0.397 \\
\hline SLEDAI & $16.72 \pm 7.95$ & $8.93 \pm 6.90$ & 0.044 \\
\hline \multicolumn{4}{|l|}{ Laboratory findings } \\
\hline Urinary protein (g/24) & $4.15(0.85-14.63)$ & $2.11(0.45-10.93)$ & $<0.001$ \\
\hline Serum creatinine $(\mathrm{mg} / \mathrm{dl})$ & $2.34(0.46-7.91)$ & $0.71(0.40-2.61)$ & $<0.001$ \\
\hline $\mathrm{P} / \mathrm{C}$ ratio $(\mathrm{mg} / \mathrm{mg})$ & $1.71(0.65-3.63)$ & $0.39(0.32-0.81)$ & $<0.001$ \\
\hline $\mathrm{BUN}(\mathrm{mmol} / \mathrm{L})$ & $7.63(2.78-61.53)$ & $4.81(1.56-45.68)$ & 0.141 \\
\hline WBC $\left(\times 10^{9} / L\right)$ & $3.66(0.41-14.94)$ & $5.63(0.63-28.97)$ & 0.0148 \\
\hline $\operatorname{PLT}\left(\times 10^{9} / \mathrm{L}\right)$ & $140.14(4.67-379.71)$ & $160.43(5.82-560.91)$ & 0.2317 \\
\hline $\mathrm{Hb}(\mathrm{g} / \mathrm{dl})$ & 7.99 (5.8-10.19) & $10.2(7.24-13.71)$ & $<0.001$ \\
\hline $\operatorname{ANA}(+)$ & 102/102 (100\%) & 87/88 (98.86) & 0.887 \\
\hline \multicolumn{4}{|l|}{ Medications } \\
\hline Steroids & $102(100 \%)$ & 88 (100\%) & \\
\hline Hydroxychloroquine & $102(100 \%)$ & 88 (100\%) & \\
\hline Cyclophosphamide & 79 (77.45\%) & $0(0 \%)$ & \\
\hline Azathioprine & $56(54.90 \%)$ & $16(18.18 \%)$ & \\
\hline Methotrexate & 37 (36.27\%) & 42 (47.72\%) & \\
\hline
\end{tabular}



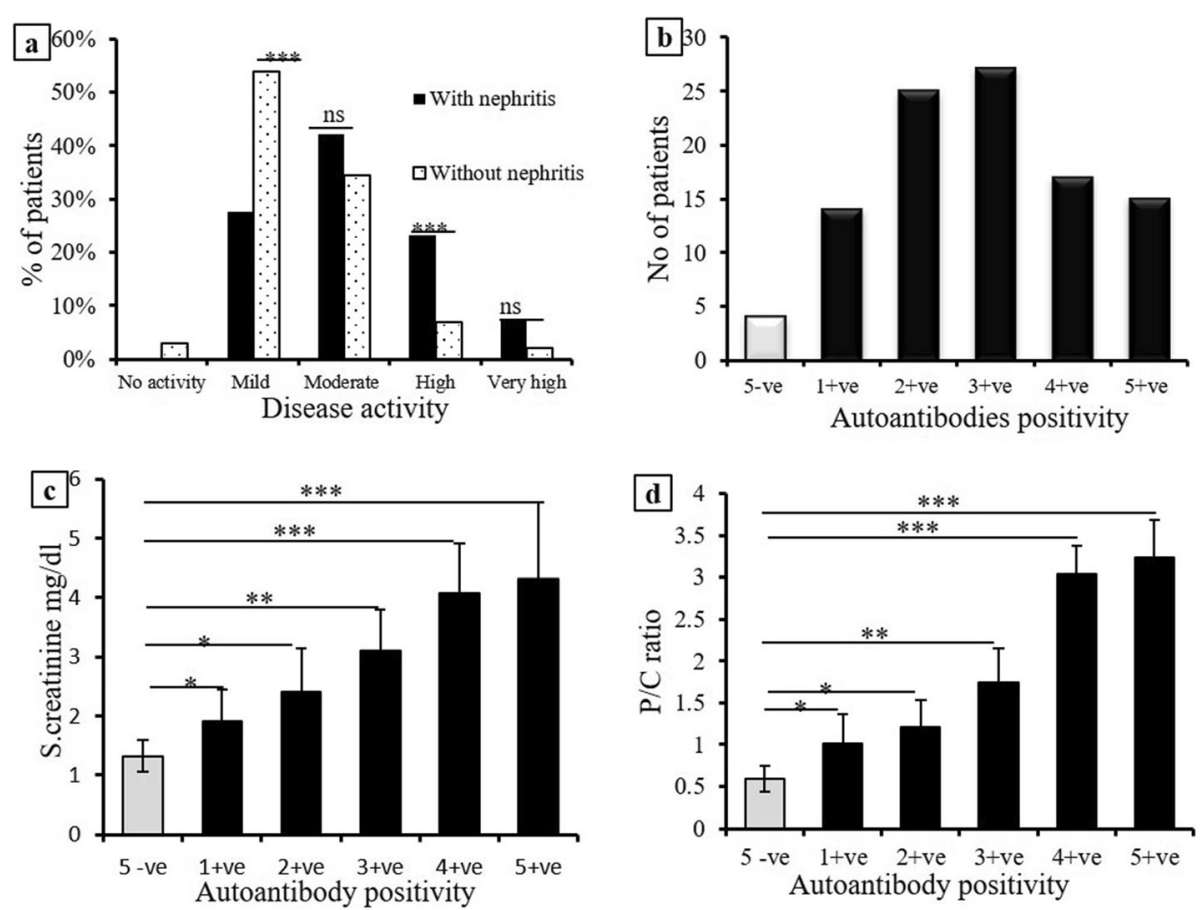

Fig. 1 Co-positivity of the five autoantibodies in patients with nephritis and its relation to disease activity, serum creatinine, and P/C ratio. (a) Comparison of the disease activity in patients with and without nephritis. (b) Number of LN patients who are negative for all or positive for one, two, three, four, and five autoantibodies. (c and $\mathbf{d}$ ) Are comparison of serum creatinine and P/C ratio among groups of five negative autoantibodies and one, two, three, four, and five positive autoantibodies. ${ }^{*} p<0.05 ;{ }^{* *} p<0.01 ;{ }^{* * *} p<0.001$; ns, nonsignificant difference; - ve, negative; +ve, positive

Table 2 Comparison of the ANA profile in SLE patients with and without nephritis regarding autoantibody positivity

\begin{tabular}{llll}
\hline Autoantibody & SLE with nephritis $(n=102)$ & SLE without nephritis $(n=88)$ & $p$ value \\
\hline Anti-dsDNA & $66(64.70 \%)$ & $23(26.1 \%)$ & $<0.001$ \\
Anti-nucleosome & $59(57.84 \%)$ & $26(29.5 \%)$ & $<0.001$ \\
Anti-histone & $46(45.09 \%)$ & $18(20.45 \%)$ & $<0.001$ \\
Anti-RO52 & $38(37.25 \%)$ & $20(22.72 \%)$ & $<0.05$ \\
Anti-Ro60 & $42(41.17 \%)$ & $18(20.45 \%)$ & $<0.05$ \\
Anti-Smith & $23(22.5 \%)$ & $20(22.7 \%)$ & 0.647 \\
Anti-RNP & $18(17.64 \%)$ & $16(18.18 \%)$ & 0.732 \\
Anti-La & $11(10.8 \%)$ & $18(20.5 \%)$ & 0.194 \\
Anti-SCL70 & $1(1 \%)$ & $4(4.5 \%)$ & 0.180 \\
Anti-RNA polymerase & $2(2 \%)$ & $7(7.95 \%)$ & 0.096 \\
Anti-Ku & $4(3.9 \%)$ & $3(3.40 \%)$ & 0.705 \\
Anti-PMSCL100 & $10(9.8 \%)$ & $7(7.95 \%)$ & 0.467 \\
Anti-SMRNP & $19(18.62 \%)$ & $12(13.63 \%)$ & 0.209 \\
Anti-MI2 & $0(0 \%)$ & $1(1.1 \%)$ & 1.00 \\
Anti-Jo1 & $4(3.9 \%)$ & $2(2.3 \%)$ & 0.414 \\
Anti-CENPA & $4(3.9 \%)$ & $2(2.3 \%)$ & 0.414 \\
Anti-PCNA & $6(5.88 \%)$ & $2(2.3 \%)$ & 0.157 \\
Anti-ribosome & $23(22.54 \%)$ & $19(21.59 \%)$ & 0.537 \\
\hline
\end{tabular}


From these autoantibodies, anti-dsDNA, anti-nucleosome, anti-histone, and anti-Ro60 were nearly twice more detected in patients with nephritis in comparison to those without nephritis $(p<0.001)$, and there was a significantly high frequency of positivity for anti-Ro52 $(p=0.018)$ in LN patients. Anti-MI2 was the least detected autoantibody.

\section{Co-positivity of the five autoantibodies in patients with nephritis and its correlation with disease activity}

As shown in Fig. 1b, out of 102 patients with nephritis, 4 were negative for the five autoantibodies, 14 were positive for only one, 25 were positive for two, 27 were positive for three, 17 were positive for four, and 15 were positive for the five autoantibodies. The effect of copositivity of the five autoantibodies, on kidney function is shown in Fig. 1c. By comparing the serum creatinine level in positive and negative groups, we found a high significant difference between all studied groups. The same pattern was found in the $\mathrm{P} / \mathrm{C}$ ratio as shown in Fig. 1d. As listed in Table 3, by comparing the mean rank of SLEDAI in relation to the autoantibody positivity, it was more significantly higher $(p=0.027)$ in patients with one positive autoantibody than patients with five negative autoantibodies. By increasing the number of positivity, the mean rank of SLEDAI increased in most of the groups except in one positive when compared to two positive, also in four positive when compared to five positive patients. In Table 4 , the antidsDNA showed a significant positive correlation with anti-nucleosome, anti-histone, and anti-Ro60 autoantibodies $(r=0.491, p<0.001),(r=0.245, p=0.014)$, and $(r=0.221, p=0.026)$ respectively, however, negatively correlated with anti-RO52 but this correlation was not significant $(r=-0.123, p=0.216)$. Anti-nucleosome showed a significant positive correlation with antihistone $(r=0.257, p=0.010)$; however, it is negatively

Table 3 Effect of the co-positivity of the five autoantibodies on SLEDAI

\begin{tabular}{lll}
\hline Group & Mean rank & $p$ value \\
\hline One positive vs all negative & 11.68 vs 5.30 & $\mathbf{0 . 0 2 7}$ \\
One positive vs two positive & 21.14 vs 23.13 & 0.630 \\
One positive vs three positive & 13.71 vs 21.67 & $\mathbf{0 . 0 3 9}$ \\
One positive vs four positive & 9.82 vs 20.47 & $\mathbf{0 . 0 0 1}$ \\
One positive vs five positive & 9.18 vs 17.15 & $\mathbf{0 . 0 0 6}$ \\
Two positive vs three positive & 28.52 vs 29.54 & $\mathbf{0 . 8 1 6}$ \\
Two positive vs four positive & 18.30 vs 33.06 & $\mathbf{0 . 0 0 0}$ \\
Two positive vs five positive & 17.48 vs 29.55 & $\mathbf{0 . 0 0 5}$ \\
Three positive vs four positive & 17.91 vs 28.91 & $\mathbf{0 . 0 0 5}$ \\
Three positive vs five positive & 16.56 vs 25.60 & $\mathbf{0 . 0 2 4}$ \\
Four positive vs five positive & 13.84 vs 12.95 & $\mathbf{0 . 7 7 1}$ \\
\hline
\end{tabular}

correlated with anti-RO52 but this correlation was also not significant $(r=-0.099, p=0.318)$. Anti-histone showed a significant positive correlation with anti-RO52 $(r=0.205, p=0.039)$. The anti-dsDNA, antinucleosome, anti-histone, and anti-Ro60 were significantly correlated with SLEDAI; however, antiRo52 was not correlated with SLEDAI as shown in Table 4.

\section{Distribution of the five autoantibodies and their relationship with histopathological classification in LN patients}

To test whether there is an association between the positivity of the five autoantibodies and histopathological classification of LN, as shown in Fig. 2, data for all positive patients revealed that patients with class-IV nephritis showed the highest frequency of positivity for the five autoantibodies followed by class-III. Class-IV had $40.9 \%, 38.98 \%, 45.65 \%, 38.09 \%$, and $28.94 \%$ of the positive patients for anti-dsDNA, anti-nucleosome, antihistone, anti-R060, and anti-Ro52 respectively, while it was $25.75 \%, 22.03 \%, 21.73 \%, 14.28 \%$, and $15.78 \%$ for class-III. In class-III LN, we found that both of antiRo52 and anti-Ro60 showed a significant $(p<0.05)$ lower frequency in comparison to anti-dsDNA, while in class-IV only anti-Ro52 showed a significantly $(p<0.01)$ lower frequency in comparison to anti-dsDNA.

\section{Receiver operating characteristic curve for detection of sensitivity and specificity of autoantibodies}

Data of ROC curve analysis as shown in Fig. 3 exhibited that anti-nucleosome had the highest area under the curve (AUC), 0.943 with $91.5 \%$ sensitivity and $93 \%$ specificity at cutoff $13 \mathrm{U} / \mathrm{ml}$ followed by anti-dsDNA with AUC was 0.939 with $83.3 \%$ sensitivity and $94.4 \%$ specificity at cut off $15 \mathrm{U} / \mathrm{ml}$ then anti-histone with AUC was 0.824 with $73.8 \%$ sensitivity and $80 \%$ specificity at a cutoff $10 \mathrm{U} / \mathrm{ml}$, while AUC was 0.796 for the anti-Ro60 with a $69.2 \%$ sensitivity and $71.4 \%$ specificity at cutoff $13 \mathrm{U} /$ $\mathrm{ml}$. On the other hand, anti-Ro52 had the lowest AUC 0.740 with $65.7 \%$ sensitivity and $73.1 \%$ specificity at a cut off $14 \mathrm{U} / \mathrm{ml}$.

\section{Discussion}

The presence of autoantibodies is one of SLE features and more than 200 autoantibodies were detected in SLE patients; however, less than $10 \%$ of these autoantibodies are commonly used nowadays [20,21]. Recently, there is a huge attention to the use of autoantibodies as a predictor of disease progression in early SLE, hoping to limit the morbidity and mortality [22]. Guthridge et al. concluded that studying the ANA profile may have a prognostic value in SLE patients [23]. On studying the ANA profile in our patients, we found that anti-dsDNA, anti-nucleosome, anti-histone, anti-Ro60, and anti-Ro52 
Table 4 Correlation between autoantibodies and disease activity. Kendall's tau-b was used for calculation of the correlation, where the number of LN patients was 102

\begin{tabular}{|c|c|c|c|c|c|c|c|}
\hline & & Anti-dsDNA & Anti-nucleosome & Anti-histone & Anti-RO60 & Anti-RO52 & SLEDAI \\
\hline \multirow[t]{2}{*}{ Anti-dsDNA } & r & 1.000 & $0.491^{* *}$ & $0.245^{*}$ & $0.221^{*}$ & -0.123 & $0.233^{* *}$ \\
\hline & $p$ & & 0.000 & 0.014 & 0.026 & 0.216 & 0.005 \\
\hline \multirow[t]{2}{*}{ Anti-nucleosome } & r & $0.491^{* *}$ & 1.000 & $0.257^{* *}$ & 0.105 & -0.099 & $0.182^{*}$ \\
\hline & $p$ & 0.000 & & 0.010 & 0.293 & 0.318 & 0.029 \\
\hline \multirow[t]{2}{*}{ Anti-histone } & r & $0.245^{*}$ & $0.257^{* *}$ & 1.000 & 0.032 & $0.205^{*}$ & $0.198^{*}$ \\
\hline & $p$ & 0.014 & 0.010 & & 0.746 & 0.039 & 0.017 \\
\hline \multirow[t]{2}{*}{ Anti-RO60 } & r & $0.221^{*}$ & 0.105 & 0.032 & 1.000 & 0.061 & $0.188^{*}$ \\
\hline & $p$ & 0.026 & 0.293 & 0.746 & & 0.543 & 0.024 \\
\hline \multirow[t]{2}{*}{ Anti-RO52 } & r & -0.123 & -0.099 & $0.205^{*}$ & 0.061 & 1.000 & 0.070 \\
\hline & $p$ & 0.216 & 0.318 & 0.039 & 0.543 & & 0.400 \\
\hline
\end{tabular}

$r$ correlation coefficient

*Correlation is significant at $p<0.05$

${ }^{* *}$ Correlation is highly significant at $p<0.01$

autoantibodies were implicated with the highest frequency, so we evaluated the alleged role for their use as biomarkers for SLE and their relationship with disease activity and renal affection. On comparing the frequency of anti-dsDNA, anti-nucleosome, anti-histone, antiRo60, and anti-Ro52 autoantibodies between patients with nephritis and those without nephritis, we found a very high significant difference regarding, anti-dsDNA, anti-nucleosome, and anti-histones and this agrees with Sui et al. and Yang et al. [24, 25], and a high significant difference regarding anti-Ro60 and anti-Ro52, this in agreement with Kerboua et al. and Mahmoudi et al. [26, 27]. It is reported that anti-Ro60 is detected in SLE and Sjogren's syndrome with a much higher percentage in cutaneous lupus erythematosus while anti-Ro52 is associated with more diseases as interstitial lung disease, congenital heart block, neoplastic diseases and infections [28-30].

Our findings confirmed that increased number of autoantibody positivity was accompanied with increased mean rank of the SLEDAI and this was clear when we compared the mean rank of patients who are positive for only one autoantibody with that of patients who are negative for the five antibodies and also when we compared all other groups. Despite the elevated mean rank of SLEDA in the group of patients who were positive for the five autoantibodies, there is no significant difference between four positive and five positive groups. Our

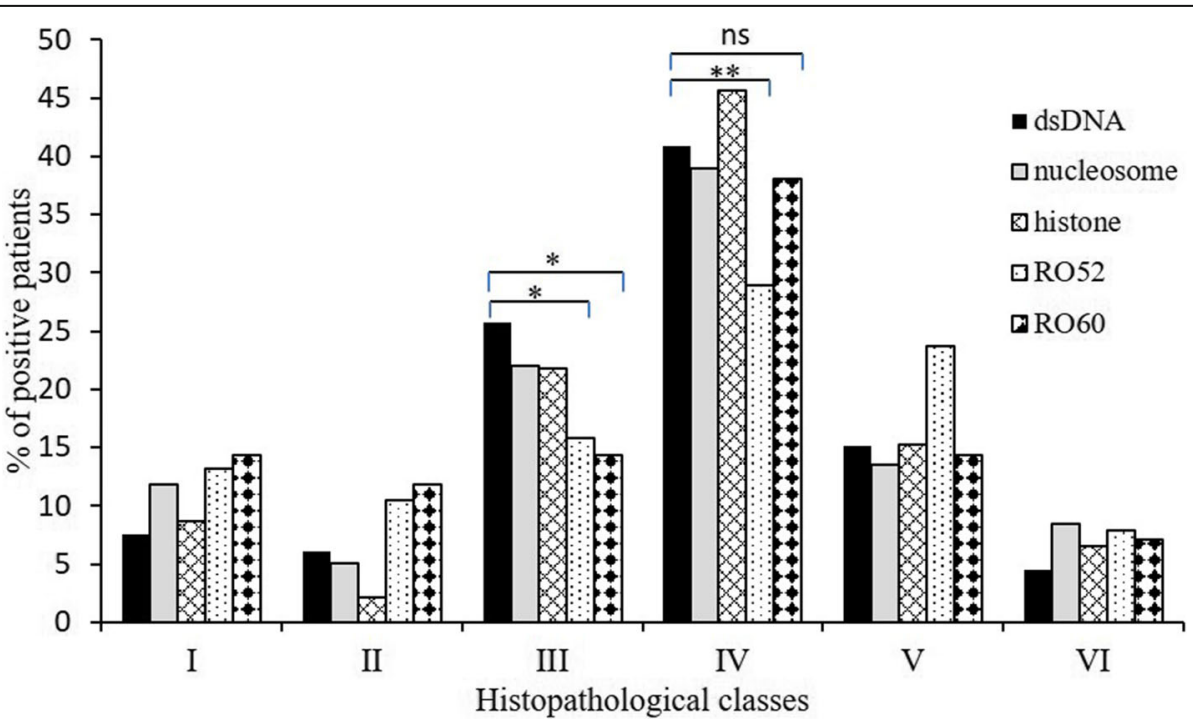

Fig. 2 Percentage of the positivity of the five autoantibodies in each class of LN. The highest percentage for all autoantibodies was found in class-IV followed by class-III. * $p<0.05 ;{ }^{* *} p<0.01$; ns, nonsignificant difference 

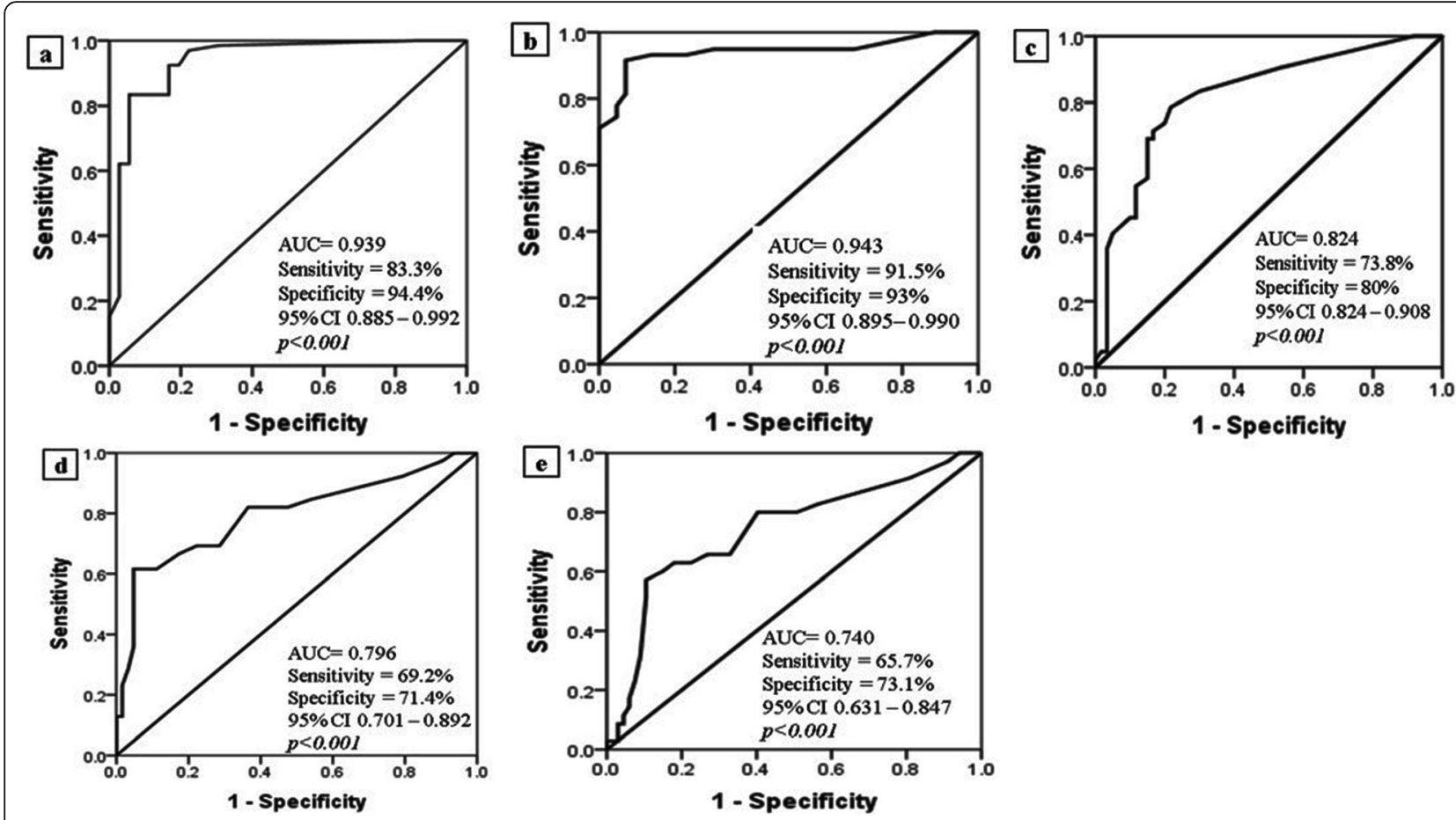

Fig. 3 Receiver operating curve. (a) anti-dsDNA, (b) anti-nucleosome, (c) anti-histone, (d) anti-Ro60, (e) anti-Ro52

finding agrees with the data previously reported by Van der Vlag and Waldman that autoantibodies have a marked role in disease manifestation and are implicated in the active stage of the disease [31, 32], Mahmoudi et al. found that anti-dsDNA and anti-histone were correlated positively with disease activity [27], also Gheita et al. reported that anti-dsDNA level is found to be significantly higher in active patients [33], Suleiman et al. reported that anti-nucleosome has a strong association with the disease activity [34]. Our finding revealed that anti-Ro60 was correlated with anti-dsDNA and SLEDAI while anti-Ro52 was negatively correlated with antidsDNA that agrees with Menendez et al. [35] and negatively correlated with anti-nucleosome, and also there was no correlation between anti-Ro52 and SLEDAI. This may reinforce the previous studies on anti-Ro52 which reported that its role was more manifested in other diseases such as interstitial lung disease, congenital heart block, neoplastic diseases, and infections [28, 36]. Our finding is in contrast with Robbin et al. who found that patients with isolated anti-Ro52 antibodies had more manifested clinical manifestations and renal affection [37].

Autoantibodies are known to increase the risk of organ involvement, and have an essential role in the pathogenesis of renal involvement in SLE [38, 39]. Antinucleosome antibody can bind the glomerular basement membrane in vivo and induce proteinuria $[40,41]$. Anti-
dsDNA is playing an important role in the initial events involved in the pathogenesis of the disease [32]. Nawata et al. and Wang et al. reported that high titer of antidsDNA is associated with hematuria, proteinuria, elevated serum creatinine, nephrotic syndrome, and hypertension [42, 43]. Rasten et al. reported that antidsDNA significantly increased in male patients with lupus nephritis [44]. Despite several previous studies pointed to the importance of anti-dsDNA in SLE, little data showed the combined effect of anti-dsDNA, antinucleosome, and anti-histone antibodies on kidney affection. According to our knowledge, no studies have been reported showing the combined impact of the five autoantibodies on renal affection and their correlation with histopathological classification. From ROC curve analysis, we found that both anti nucleosome and antidsDNA had high sensitivity and specificity for LN with anti-nucleosome had a higher sensitivity than antidsDNA, and this in agreement with Abdel Gawad et al. and Gutiérrez-Adrianzén et al. [45, 46]. While antihistone, anti-Ro60, and anti-Ro52 showed low sensitivity and specificity for LN. Sui et al. studied the simultaneous positivity of anti-DNA, anti-nucleosome, and antihistone antibodies, and they found a strong association between the positivity and renal disease activity [24]. Despite the studies on the association between antiRo52 and anti-Ro60 with certain diseases, the results of these studies are contradicting. Our finding revealed that 
the impact of the co-positivity of the five autoantibodies on the renal function was obvious since we found a significant increase in serum creatinine level and $\mathrm{P} / \mathrm{C}$ ratio in co-positive $\mathrm{LN}$ patients as a marker for the severity of the renal affection, similar results were reported by Yang et al. [25].

Autoantibodies induce immune-mediated kidney diseases [47]. Pathology of lupus nephritis involves antibody binding to several intrarenal autoantigens rather than immune complexes deposition [48], so the production of autoantibodies may play a role in the development of nephropathy as reported by Sui et al. and Yang et al. [24, 25]. According to our findings, class-III and class-IV (focal and diffuse lupus nephritis) had the highest frequency of positivity for the five autoantibodies, where $66.6 \%$ of the positive cases for anti-dsDNA, 61\% for anti-nucleosome, $67.38 \%$ for anti-histone, $52.37 \%$ for anti-Ro60, and $44.72 \%$ for anti-Ro52 located in class-III and IV collectively. Our finding for anti-dsDNA, antinucleosome, and anti-histone agrees with Sui et al. [24], but Ahmed et al. reported that renal involvements were found in $66.1 \%$ of patients and class-III was the dominant histological lesion [49]. Our results for anti-Ro52 and anti-Ro60 agree with Kerboua et al. who reported that LN patients have increased circulating immune complexes associated with anti-SSA, anti-SSB, anti-Sm, and anti-C1q [26]. Despite the humble frequency of both anti-Ro60 and anti-Ro52 in class-III LN, however, 38\% of the anti-Ro60 positive cases and $28.94 \%$ of the antiRo52 positive cases were found in class-IV LN.

Autoantibodies against dsDNA, histone and nucleosome have been reported to be linked to the development of nephritis in SLE patients [20]. We supposed that the copositivity of the five autoantibodies may play a role in the development of LN and may predict more severe disease activity and renal affection; however, our data revealed that despite the considerable high frequency of anti-Ro52 in $\mathrm{LN}$ patients but it seems that its effect on disease activity, renal parameters, and pathological changes was not manifested, and it has a low specificity; this may explain the insignificant difference on comparing the mean rank of SLEDAI between one positive vs two positive, two positive vs three, and four positive vs five positive groups. Our hypothesis was confirmed by finding that the reactivity of these five autoantibodies was correlated with each other and also with SLEDAI except for anti-Ro52 which had no correlation with SLEDAI and negatively but not significantly correlated with anti-dsDNA and anti-nucleosome. Our data, supposed the implication of the co-positivity of anti-dsDNA, anti-nucleosome, anti-histone, and antiRo60 as risk factors for the development of proliferative nephritis and for more active disease, so their detection may be considered as a prognostic marker for disease progression.

\section{Conclusion}

Detection of anti-dsDNA, anti-nucleosome, anti-histone, and anti-Ro60 in SLE patients may be important for predicting disease progression and kidney affection. Moreover, anti-nucleosome and anti-dsDNA show high sensitivity and specificity for lupus nephritis, thus patients with four to five positive autoantibody panels should be kept under close monitoring as they may warrant, considering aggressive therapy to control their disease and prevent renal damage.

Abbreviations

SLE: Systemic lupus erythematosus; LN: Lupus nephritis; ROC: Receiver operating curve; ANA: Anti-nuclear antibodies; SLEDAl: Systemic lupus erythematosus disease activity index; AUC: Area under the curve

\section{Acknowledgements}

The authors acknowledge the effort exerted by members of the Pathology Department, Faculty of Medicine, Sohag University, for the histopathological assessment part of this study.

\section{Authors' contributions}

The conceptualization of this study, selection and diagnosis of patients, and the collection of data were performed by S.A.E. The manuscript was written and revised, and the data interpretation was performed by both S.A. E. and O.M.M. All authors have read and approved the manuscript.

\section{Funding}

This study had no funding from any resource.

\section{Availability of data and materials}

The data of the current study are available from the corresponding author on reasonable request.

\section{Ethics approval and consent to participate}

This study was carried out in accordance with the ethical standards laid down in the Helsinki Declaration of 1975 and its later amendments in 2000, and approved by The Medical Research Ethics Committee, Faculty of Medicine-Sohag University under the reference number IBR\#S20-134. All patients included in this study gave written informed consent to participate in this research.

\section{Consent for publication}

All patients included in this research gave written informed consent to publish the data contained within this study.

\section{Competing interests}

None

\section{Author details}

'Department of Rheumatology and Rehabilitation, Faculty of Medicine, Sohag University, Sohag 82524, Egypt. ${ }^{2}$ Department of Biochemistry, Faculty of Pharmacy, Al-Azhar University, Assiut Branch, Assiut 71524, Egypt.

Received: 30 June 2020 Accepted: 25 August 2020

Published online: 12 October 2020

\section{References}

1. Rekvig OP (2015) Anti-dsDNA antibodies as a classification criterion and a diagnostic marker for systemic lupus erythematosus: critical remarks. Clin Exp Immunol 179:5-10

2. Melderis S, Wiech T, Iking-Konert C, Steinmetz OM (2018) Lupus nephritis. Z Rheumatol 77:593-608

3. Ichinose K, Kitamura M, Sato S, Eguchi M, Okamoto M, Endo Y, Tsuji S, Takatani A, Shimizu T, Umeda M et al (2019) Complete renal response at 12 months after induction therapy is associated with renal relapsefree rate in lupus nephritis: a single-center, retrospective cohort study. Lupus 28:501-509 
4. Nachman PH (2018) Repeat kidney biopsy for lupus nephritis: an important step forward. Kidney Int 94:659-661

5. Hauksdottir GS, Jonsson T, Mellbye OJ (2001) Antinuclear antibody (ANA) profile testing of ANA- positive patients. Clin Rheumatol 20:157-158

6. Flechsig A, Rose T, Barkhudarova F, Strauss R, Klotsche J, Dähnrich C, Schlumberger W, Enghard P, Burmester GR, Hiepe F et al (2017) What is the clinical significance of anti-Sm antibodies in systemic lupus erythematosus? A comparison with anti-dsDNA antibodies and C3. Clin Exp Rheumatol 35: 598-606

7. Leuchten N, Hoyer A, Brinks R, Schoels M, Schneider M, Smolen J, Johnson SR, Daikh D, Dörner T, Aringer M et al (2018) Performance of antinuclear antibodies for classifying systemic lupus erythematosus: a systematic literature review and meta-regression of diagnostic data. Arthritis Care Res 70:428-438

8. Li T, Prokopec SD, Morrison S, Lou W, Reich H, Gladman D, Urowitz M, Scholey J, Fortin PR, Boutros PC et al (2015) Anti-nucleosome antibodies outperform traditional biomarkers as longitudinal indicators of disease activity in systemic lupus erythematosus. Rheumatology (Oxford, England) 54:449-457

9. Simón JA, Cabiedes J, Ortiz E, Alcocer-Varela J, Sánchez-Guerrero J (2004) Anti-nucleosome antibodies in patients with systemic lupus erythematosus of recent onset. Potential utility as a diagnostic tool and disease activity marker. Rheumatology (Oxford, England) 43:220-224

10. Bigler C, Lopez-Trascasa M, Potlukova E, Moll S, Danner D, Schaller M, Trendelenburg M (2008) Antinucleosome antibodies as a marker of active proliferative lupus nephritis. Am J Kidney diseases 51:624-629

11. González C, Garcia-Berrocal B, Herráez O, Navajo JA, González-Buitrago JM (2004) Anti-nucleosome, anti-chromatin, anti-dsDNA and anti-histone antibody reactivity in systemic lupus erythematosus. Clin Chem Lab Med 42: 266-272

12. Lee AYS (2017) A review of the role and clinical utility of anti-Ro52/TRIM21 in systemic autoimmunity. Rheumatol Int 37:1323-1333

13. Fujimoto M, Shimozuma M, Yazawa N, Kubo M, Ihn H, Sato S, Tamaki T, Kikuchi K, Tamaki K (1997) Prevalence and clinical relevance of 52-kDa and 60-kDa Ro/SS-A autoantibodies in Japanese patients with systemic sclerosis. Ann Rheum Dis 56:667-670

14. Menéndez A, Gómez J, Caminal-Montero L, Díaz-López JB, CabezasRodríguez I, Mozo L (2013) Common and specific associations of anti-SSA Ro60 and anti-Ro52/TRIM21 antibodies in systemic lupus erythematosus. Sci World J 2013:832789

15. Font J, Cervera R (1993) 1982 revised criteria for classification of systemic lupus erythematosus - ten years later. Lupus 2:339-341

16. Bombardier C, Gladman DD, Urowitz MB, Caron D, Chang CH (1992) Derivation of the SLEDAI. A disease activity index for lupus patients. The Committee on Prognosis Studies in SLE. Arthritis Rheum 35:630-640

17. Peng $X$, Tang J, Wu Y, Yang B, Hu J (2014) Novel method for ANA quantitation using IIF imaging system. J Immunol Methods 404:52-58

18. Kern P, Kron M, Hiesche K (2000) Measurement of antinuclear antibodies: assessment of different test systems. Clin Diagn Lab Immunol 7:72-78

19. $\mathrm{Ma} \mathrm{H}$, Bandos Al, Gur D (2015) On the use of partial area under the ROC curve for comparison of two diagnostic tests. Biomet J Biometrische Zeitschrift 57:304-320

20. Dieker J, Berden JH, Bakker M, Briand JP, Muller S, Voll R, Sjowall C, Herrmann M, Hilbrands LB, van der Vlag J (2016) Autoantibodies against modified histone peptides in SLE patients are associated with disease activity and lupus nephritis. PLoS One 11:e0165373

21. An Y, Zhou Y, Bi L, Liu B, Wang H, Lin J, Xu D, Wang M, Zhang J, Wang Y et al (2019) Combined immunosuppressive treatment (CIST) in lupus nephritis: a multicenter, randomized controlled study. Clin Rheumatol 38: 1047-1054

22. Haddon DJ, Diep VK, Price JV, Limb C, Utz PJ, Balboni I (2015) Autoantigen microarrays reveal autoantibodies associated with proliferative nephritis and active disease in pediatric systemic lupus erythematosus. Arthritis Res Ther 17:162

23. Guthridge CJ, Gross T, Quintero M, Kheir JM, Levin J, Bourn RL, Khan S, Peercy M, Saunkeah B, Guthridge JM et al (2020) Expanded autoantibody profiles for subsetting of Native American, African American, and European American patients with systemic lupus erythematosus. 2:415-423

24. Sui M, Lin Q, Xu Z, Han X, Xie R, Jia X, Guo X, Zhang W, Guan X, Ren H (2013) Simultaneous positivity for anti-DNA, anti-nucleosome and anti- histone antibodies is a marker for more severe lupus nephritis. J Clin Immunol 33:378-387

25. Yang J, Xu Z, Sui M, Han J, Sun L, Jia X, Zhang H, Han C, Jin X, Gao F et al (2015) Co-positivity for anti-dsDNA, -nucleosome and -histone antibodies in lupus nephritis is indicative of high serum levels and severe nephropathy. PLoS One 10:e0140441

26. Kerboua K, Chaib S, Haiba F (2016) Association between anti-Ro 60 kDa (SSA) autoantibodies and hypocomplementemia in systemic lupus erythematosus patients from Algiers prefectures. Egyptian Rheumatologist 38:171-176

27. Mahmoudi M, Rastin M, Sahebari M, Zamani S, Tabasi N (2017) Autoantibody profile, disease activity and organ involvement in Iranian systemic lupus erythematosus patients. Rheumatol Res 2:11-16

28. Ghillani P, Andre C, Toly C, Rouquette AM, Bengoufa D, Nicaise P, Goulvestre C, Gleizes A, Dragon-Durey MA, Alyanakian MA et al (2011) Clinical significance of anti-Ro52 (TRIM21) antibodies non-associated with anti-SSA $60 \mathrm{kDa}$ antibodies: results of a multicentric study. Autoimmun Rev 10:509-513

29. Menéndez A Gómez J, Escanlar E, Caminal-Montero L, Mozo L (2013) Clinical associations of anti-SSA/Ro60 and anti-Ro52/TRIM21 antibodies: diagnostic utility of their separate detection. Autoimmunity 46:32-39

30. Menor Almagro R, Jurado Roger A, Rodríguez Gutiérrez FJ, Solís Díaz R, Cardiel MH, Salaberri Maestrojuan JJ (2016) Association of anti-Ro52, antiRo60 and anti-La antibodies with diagnostic, clinical and laboratory features in a referral hospital in Jerez, Spain. Reumatologia Clinica 12:256-262

31. van der Vlag J, Berden JH (2011) Lupus nephritis: role of antinucleosome autoantibodies. Semin Nephrol 31:376-389

32. Waldman M, Madaio MP (2005) Pathogenic autoantibodies in lupus nephritis. Lupus 14:19-24

33. Gheita TA, Abaza NM, Hammam N, Mohamed AAA, El G II, Eissa AH (2018) Anti-dsDNA titre in female systemic lupus erythematosus patients: relation to disease manifestations, damage and antiphospholipid antibodies. Lupus 27:1081-1087

34. Suleiman S, Kamaliah D, Nadeem A, Naing NN, Che Maraina CH (2009) Antinucleosome antibodies as a disease activity marker in patients with systemic lupus erythematosus. Int I Rheum Dis 12:100-106

35. Menendez A, Gomez J, Caminal-Montero L, Diaz-Lopez JB, CabezasRodriguez I, Mozo L (2013) Common and specific associations of anti-SSA/ Ro60 and anti-Ro52/TRIM21 antibodies in systemic lupus erythematosus. TheScientificWorldJournal 2013:832789

36. Fritsch C, Hoebeke J, Dali H, Ricchiuti V, Isenberg DA, Meyer O, Muller S (2006) $52-k D a$ Ro/SSA epitopes preferentially recognized by antibodies from mothers of children with neonatal lupus and congenital heart block. Arthritis Res Ther 8:R4

37. Robbins A, Hentzien M, Toquet S, Didier K, Servettaz A, Pham BN, Giusti D (2019) Diagnostic utility of separate anti-Ro60 and anti-Ro52/TRIM21 antibody detection in autoimmune diseases. Front Immunol 10:444

38. Hiepe F, Radbruch A (2016) Plasma cells as an innovative target in autoimmune disease with renal manifestations. Nat Rev Nephrol 12:232-240

39. Stummvoll G, Aringer M, Handisurya A, Derfler K (2017) Immunoadsorption in autoimmune diseases affecting the kidney. Semin Nephrol 37:478-487

40. Chan TM, Leung JK, Ho SK, Yung S (2002) Mesangial cell-binding anti-DNA antibodies in patients with systemic lupus erythematosus. J Am Soc Nephrol 13:1219-1229

41. Mjelle JE, Rekvig OP, Van Der Vlag J, Fenton KA (2011) Nephritogenic antibodies bind in glomeruli through interaction with exposed chromatin fragments and not with renal cross-reactive antigens. Autoimmunity 44: 373-383

42. Nawata T, Kubo M, Fujii S, Shiragami K, Ikegami T, Kobayashi S, Hisano S, Yano M (2018) Treatment of class IV lupus nephritis with mycophenolate mofetil monotherapy. Intern Med 57:2067-2070

43. Wang J, Qi YY, Chen XP, Ma L, Zhang LL, Zhao Y, Wang M (2018) Analysis of clinical risk factors in relapsed patients with class IV lupus nephritis. Exp Ther Med 15:5461-5468

44. Rastin M, Mahmoudi M, Sahebari M, Tabasi N (2017) Clinical \& immunological characteristics in systemic lupus erythematosus patients. Indian J Med Res 146:224-229

45. Abdel Gawad ER, Mansour Al, Abdel Aziz YA, Soliman AF, Fawzy RM (2014) Role of anti-nucleosome antibodies in the diagnosis of systemic lupus erythematosus and as a marker for lupus nephropathy. Egypt J Immunol 21: $57-65$ 
46. Gutiérrez-Adrianzén OA, Koutouzov S, Mota RM, das Chagas Medeiros MM, Bach JF, de Holanda Campos H (2006) Diagnostic value of anti-nucleosome antibodies in the assessment of disease activity of systemic lupus erythematosus: a prospective study comparing anti-nucleosome with antidsDNA antibodies. J Rheumatol 33:1538-1544

47. Tecklenborg J, Clayton D, Siebert S (2018) The role of the immune system in kidney disease. 192:142-150

48. Lech M, Anders HJ (2013) The pathogenesis of lupus nephritis. J Am Soc Nephrol 24:1357-1366

49. Ahmed N, Shigidi M, Al Agib AN, Abdelrahman H, Taha E (2017) Clinical features and antinuclear antibodies profile among adults with systemic lupus erythematosus and lupus nephritis: a cross-sectional study. Pan Afr Med J 27:114

\section{Publisher's Note}

Springer Nature remains neutral with regard to jurisdictional claims in published maps and institutional affiliations.

\section{Submit your manuscript to a SpringerOpen ${ }^{\circ}$ journal and benefit from:}

- Convenient online submission

Rigorous peer review

- Open access: articles freely available online

- High visibility within the field

- Retaining the copyright to your article

Submit your next manuscript at $\boldsymbol{\nabla}$ springeropen.com 